\title{
The impact of arm position and pulse pressure on the validation of a wrist-cuff blood pressure measurement device in a high risk population
}

This article was published in the following Dove Press journal:

International Journal of General Medicine

10 March 2010

Number of times this article has been viewed

\author{
Ali Reza Khoshdel ${ }^{1,2}$ \\ Shane Carney ${ }^{2}$ \\ Alastair Gillies ${ }^{2}$ \\ 'Faculty of Medicine, Aja University \\ of Medical Science, Tehran, Iran; \\ ${ }^{2}$ John Hunter Hospital, Faculty of \\ Health, The University of Newcastle, \\ Newcastle, NSW, Australia
}

\begin{abstract}
Despite the increasing popularity of blood pressure (BP) wrist monitors for self-BP measurement at home, device validation and the effect of arm position remains an issue. This study focused on the validation of the Omron HEM-609 wrist BP device, including an evaluation of the impact of arm position and pulse pressure on BP measurement validation. Fifty patients at high risk for cardiovascular disease were selected (age $65 \pm 10$ years). Each patient had two measurements with a mercury sphygmomanometer and three measurements with the wrist BP device (wrist at the heart level while the horizontal arm supported [HORIZONTAL], hand supported on the opposite shoulder [SHOULDER], and elbow placed on a desk [DESK]), in random order. The achieved systolic BP (SBP) and diastolic BP (DBP) wrist-cuff readings were compared to the mercury device and the frequencies of the readings within 5, 10, and $15 \mathrm{mmHg}$ of the gold standard were computed and compared with the British Hypertension Society (BHS) and Association for the Advancement of Medical Instrumentation (AAMI) protocols. The results showed while SBP readings with HORIZONTAL and SHOULDER positions were significantly different from the mercury device (mean difference $=7.1$ and $13.3 \mathrm{mmHg}$, respectively; $P<0.05$ ), the DESK position created the closest reading to mercury (mean difference $=3.8, P>0.1$ ). Approximately $71 \%$ of SBP readings with the DESK position were within $\pm 10 \mathrm{mmHg}$, whereas it was $62.5 \%$ and $34 \%$ for HORIZONTAL and SHOULDER positions, respectively. Wrist DBP attained category $\mathrm{D}$ with BHS criteria with all three arm positions. Bland-Altman plots illustrated that the wrist monitor systematically underestimated SBP and DBP values. However a reading adjustment of 5 and $10 \mathrm{mmHg}$ for SBP and DBP (DESK position) resulted in improvement with $75 \%$ and $77 \%$ of the readings being within $10 \mathrm{mmHg}$ (grade B), respectively. AAMI criteria were not fulfilled due to heterogeneity. The findings also showed that the mismatch between the mercury and wrist-cuff systolic BP readings was directly associated with pulse pressure. In conclusion the DESK position produces the most accurate readings when compared to the mercury device. Although wrist BP measurement may underestimate BP measured compared to a mercury device, an adjustment by 5 and $10 \mathrm{mmHg}$ for SBP and DBP, respectively, creates a valid result with the DESK position. Nevertheless, considering the observed variations and the possible impact of arterial stiffness, individual clinical validation is recommended.
\end{abstract}

Keywords: blood pressure, device validation, position

\section{Introduction}

The mercury sphygmomanometer is disappearing from clinical practice partially due to the environmental disadvantages of mercury. ${ }^{1}$ This factor, combined with aneroid device calibration problems and the convenience of automated oscillometric devices for both patients and clinicians, will probably lead to the latter devices being the preferred blood pressure (BP) measurement method.
Correspondence: Ali Reza Khoshde Department of Nephrology, John Hunter Hospital, Locked Bag I, Hunter Region Mail Centre, NSW 23I0, Australia

Tel +62249214321

Fax +62 249214339

Email alikhoshdel@yahoo.com 
Automated devices are not only more convenient to use but are also less prone to human error when compared to conventional auscultatory techniques. ${ }^{2,3}$ While oscillometric devices used in clinical practice were based on upper arm brachial artery compression, the wrist radial artery oscillometric technique is now available. In contrast to upper arm oscillometric devices which are relatively accurate when compared to mercury devices, ${ }^{2}$ wrist blood pressure measurement may be subject to inaccuracies caused by peripheral vasoconstriction and incorrect limb position. ${ }^{2,4}$ However, the popularity and marketing of wrist blood pressure monitors has been increasing ${ }^{2,5-7}$ and consequently independent validation and clarification of the correct measurement technique with a wrist monitor is of great importance.

As a general rule, BP devices should be assessed for safety, accuracy, and reliability prior to marketing ${ }^{8}$ and there are several standard protocols including the US Association for the Advancement of Medical Instrumentation (AAMI) protocol, British Hypertension Society (BHS) protocol and the international protocol delivered by European Society of Hypertension (ESH). ${ }^{89}$ Unfortunately, few devices worldwide have been independently validated. ${ }^{2,4}$ In a recent ESH report reviewing manual and automated blood pressure devices, two types of wrist monitors were classified in the questionable recommendation category because of limited evidence. ${ }^{4,6}$ Likewise, The Japanese Society of Hypertension recommended that wrist-cuff devices should not be used for home BP measurement. ${ }^{10}$ The problem seems more complex with different recommendations regarding limb position during wrist-cuff blood pressure measurement. Although published recommendations insist that the wrist should be at heart level during measurement, there is no agreement on arm position. Whereas some suggest a shoulder-supported position, others recommend a horizontal arm or a desk-supported position. In addition, it is not know whether heart level can consistently be achieved in practice for self-measurement at home.

There is no study comparing the accuracy of these positions. Moreover, while most validation studies included normal healthy subjects, extrapolation of their results to the average hypertensive population in routine clinics who often have multiple comorbidities is unreasonable. Therefore, this study focused on the validation of a wrist-cuff BP monitor in a high risk population and included an evaluation of the impact of arm position and pulse pressure (PP) on the validity of the BP measurement. This study attempted to maintain the main concepts of the above mentioned protocols but expand on their flexibility.

\section{Methods}

Measurements were performed by a trained physician (AK) five times for each patient. The observer's competence was achieved prior to the validation process by reviewing the latest protocols for BP measurements and the website for BP measurement delivered by BHS. ${ }^{11}$ The subjects were seated in a quiet room and BP measurements were commenced after a 5-10 minute rest. Measurements were carried out in the following sequence: Mercury 1 (M1), three measurements with the wrist monitor (W1, W2, and W3) with a random order regarding arm position, and finally Mercury 2 (M2). A period of 30-60 seconds occurred between each measurement to avoid venous congestion. The same arm was used for all measurements. All wrist monitor measurements were recorded in its memory and the observer was blinded for these measurements.

A standard mercury sphygmomanometer was used as a reference standard. BP measured with the arm supported at heart level and the level of the manometer was at eye level, within one meter of the observer. A well maintained quality adjustable cuff was used for the measurements and Korotkoff sound phase $\mathrm{V}$ was used to determine the diastolic pressure. All readings with the mercury device were recorded to the nearest $2 \mathrm{mmHg}$.

The wrist monitor was the oscillometric Omron HEM609 (Omron, Kyoto, Japan) equipped with an IntelliSense ${ }^{\mathrm{TM}}$ technology, which eliminates the potential inaccuracies resulted from an irregular pulse and incorrect position and individualizing the inflation and deflation rate. The cuff is suitable for a $5^{1 / 4^{\prime \prime}}$ to $8 \frac{1}{2} 2^{\prime \prime}$ wrist circumference. Measurement range for the device is 0 to $280 \mathrm{mmHg}$ with pulse rate of 40 to 180 beats per minute. It was used in an optimal range of temperature and humidity.

Forty pairs of consecutive measurements were used for reliability test (on same arm), followed by a validation study. The observer examined each individual three times in three different positions with the wrist monitor: 1) with the horizontal supported arm and the wrist at heart level (HORIZONTAL); 2) with the hand on the opposite shoulder (SHOULDER); 3) with a bent elbow supported on a desk and the wrist at heart level (DESK). The readings were recorded in the device memory and were deleted before using for another patient to prevent disturbing the next measurements.

\section{Classification criteria}

The BHS protocol and the international protocol (ESH) have introduced the concept of classifying the differences between 
test and control measurements according to whether these lie within 5 (or lower), 10, or 15 (or over) $\mathrm{mmHg}$, calculated separately for systolic BP (SBP) and diastolic BP (DBP). ${ }^{8,9}$ Differences were calculated by subtracting the observer mercury measurement (M) from each wrist monitor (W) device measurements (W1, W2, and W3) individually, assuming 0 to $5 \mathrm{mmHg}$ difference to be very accurate and $>15 \mathrm{mmHg}$ very inaccurate.

\section{Accuracy criteria}

The analysis was based on how values in these bands fall cumulatively into three zones: within $5 \mathrm{mmHg}$, within $10 \mathrm{mmHg}$, within $15 \mathrm{mmHg}$. ${ }^{9}$ The grading was based on the number of differences falling into these categories, where grade $\mathrm{A}$ denotes greatest agreement with mercury and grade $\mathrm{D}$ has the worst agreement (Table 1). According to AAMI, the test device must not differ from the mercury standard by a mean difference $>5 \mathrm{mmHg}$ or a standard deviation (SD) of $>8 \mathrm{mmHg}^{4}$

\section{Recommendation criteria}

The following criteria have been used to designate devices according to accuracy. A device is classified as "recommended" if it fulfils the AAMI criteria for both SBP and DBP and received a grade A or B under BHS protocol for both SBP and DBP. A device is "not recommended" if it fails the AAMI criteria for either SBP or DBP and achieves a grade $\mathrm{C}$ or $\mathrm{D}$ for either $\mathrm{SBP}$ or DBP under the BHS protocol. ${ }^{9}$

\section{Subject selection}

For a minimum number of required samples with a wide BP range, subjects were selected from different clinical settings including outpatient diabetes, hypertension and nephrology clinics, dialysis units, as well as cardiovascular, nephrology, and gastroenterology hospital wards. Some participants were taking medications, but those with atrial fibrillation were

Table I Grading criteria used by the BHS (1993). Grades are cumulative percentage of reading; values are $\mathrm{mmHg}$

\begin{tabular}{llll}
\hline & \multicolumn{2}{l}{$\begin{array}{l}\text { Cumulative absolute difference between } \\
\text { standard and test (\%) }\end{array}$} \\
\hline Grade & $\leq \mathbf{5}$ & $\leq \mathbf{1 0}$ & $\leq \mathbf{1 5}$ \\
A & 60 & 85 & 95 \\
B & 50 & 75 & 90 \\
C & 40 & 65 & 85 \\
D & Worse than C & & \\
\hline
\end{tabular}

excluded. As most devices are less accurate in the extremely high BP range, three ranges for SBP and three ranges for DBP was proposed, with at least 11 subjects in each range, according to BHS and ESH guidelines (Table 2). ${ }^{9}$

\section{Statistical analysis}

To test the device measurement reliability (repeatability) 40 pairs of wrist-cuff measurement with DESK position (one minute apart) were performed on a variety of patients groups and healthy subjects. BP ranges were defined according to the average of M1 and M2. For purpose of validity assessment, some international guidelines don't recommend using average measurements and suggest the difference of test measurements with each gold standard. ${ }^{9}$ Accordingly, in this study both the average mercury and the individual M1 and M2 have been used for comparisons. The agreement between the $\mathrm{W}$ measurements and the average $\mathrm{M}$ measurements were tested by the Pearson's and the Spearman's correlation coefficient and each of W measurements method was compared to average M, using Student's $t$-test. Validation assessment followed BHS and AAMI criteria. Coefficient of variation was calculated by $\mathrm{SD} /$ mean for each method.

\section{Results}

Fifty subjects were recruited for the sequential measurements. After exclusion of two patients with an irregular heart beat, as well as eight SBP and seven DBP out-of-range readings, 40 valid SBP and 4 valid DBP were obtained (Table 2). The average age of subjects was $65 \pm 10$ years and $60 \%$ of the patients were male. There was no significant difference between pulse rate with each $\mathrm{W}$ measurement method, indicating similarity of the conditions for the three consecutive measurements.

\section{Reliability of the wrist-cuff device}

The mean difference between two consecutive measurements was only 0.59 and $0.77 \mathrm{mmHg}$ and the Pearson correlation coefficient was $0.94(0.91-0.96)$ and $0.80(0.77-0.83)$ for the SBP and DBP measurement.

Table 2 Blood pressure ranges

\begin{tabular}{|c|c|c|c|c|}
\hline & \multicolumn{2}{|l|}{ SBP } & \multicolumn{2}{|l|}{ DBP } \\
\hline & Range & $\mathbf{N}$ & Range & $\mathbf{N}$ \\
\hline Low & $90-129$ & 15 & $40-79$ & 13 \\
\hline Medium & $130-160$ & 13 & $80-100$ & 17 \\
\hline High & $161-180$ & 12 & $101-130$ & II \\
\hline
\end{tabular}

Abbreviations: DPB, diastolic blood pressure; SBP, systolic blood pressure. 


\section{Wrist against mercury measurements}

The DESK position produced the least measurement difference with the mercury device, both for SBP and DBP values (Table 3). However in terms of variation, BP measurements with SHOULDER position had the lowest coefficient of variation ( 0.79 and 0.60 for the SBP and DBP).

There was a strong positive correlation between $\mathrm{M}$ and W SBP and DBP with all three limb positions (Table 4). Nevertheless, paired comparison of mean BP measured by M measurements and the obtained values of the $\mathrm{W}$ measurements with each limb position revealed that while BP measurements by HORIZONTAL and SHOULDER positions significantly differed from the $\mathrm{M}(P<0.01)$, the DESK position made SBP results not significantly different from the gold standard $(P>0.1)$. The mean and $95 \%$ confidence interval $(\mathrm{CI})$ of the difference between $\mathrm{M}$ and each $\mathrm{W}$ measurements were 3.9 (95\% CI: -1.2-9.1), 7.2 (95\% CI: 2.2-12.1), and 13.2 (95\% CI: 8.7-17.7) for the DESK, HORIZONTAL, and SHOULDER positions. Nevertheless, DBP was consistently underestimated by $\mathrm{W}$ with all of the three limb positions and was significantly different compared to $\mathrm{M}(P<0.0001)$.

\section{Validation test according to BHS and AAMI}

Validation of the measurements was assessed according to BHS and AAMI protocols for 180 pairs of SBP and 196 DBP readings.

While DESK and HORIZONTAL positions reached the $\mathrm{B}$ and $\mathrm{C}$ grades for the proportion of the difference equal or below $5 \mathrm{mmHg}$, they were not matched with the criteria in higher levels of difference. Furthermore despite the acceptable mean difference between $\mathrm{W}$ and $\mathrm{M}$ with DESK position $(-3.9)$ the AAMI criteria was not achieved due to significant variation $(\mathrm{SD}=13.2)($ Table 5$)$.

Table 3 Average difference in blood pressure measurement with mercury device and wrist-cuff device with three positions

\begin{tabular}{llll}
\hline Arm position & $\begin{array}{l}\text { Mean } \\
\text { difference }\end{array}$ & $\begin{array}{l}\text { Standard } \\
\text { deviation }\end{array}$ & $\begin{array}{l}\text { Coeff. of } \\
\text { variation }\end{array}$ \\
\hline Systolic & & & \\
$\quad$ SHOULDER & 13.31 & 10.48 & 0.79 \\
HORIZONTAL & 7.09 & 13.05 & 1.84 \\
$\quad$ DESK & 3.78 & 12.02 & 3.17 \\
Diastolic & & & \\
SHOULDER & 15.97 & 9.63 & 0.60 \\
HORIZONTAL & 11.85 & 9.62 & 0.81 \\
DESK & 9.41 & 9.29 & 0.98 \\
\hline
\end{tabular}

Table 4 Pearson correlation of $M$ and $W$ measurements

\begin{tabular}{|c|c|c|c|c|}
\hline \multirow[t]{2}{*}{ Arm position } & \multicolumn{2}{|l|}{ SBP } & \multicolumn{2}{|l|}{ DBP } \\
\hline & $r$ & $P$ & $r$ & $P$ \\
\hline SHOULDER & 0.86 & $<0.000$ & 0.82 & $<0.000$ \\
\hline HORIZONTAL & 0.79 & $<0.000$ & 0.85 & $<0.000$ \\
\hline DESK & 0.83 & $<0.000$ & 0.83 & $<0.000$ \\
\hline
\end{tabular}

Abbreviations: DPB, diastolic blood pressure; SBP, systolic blood pressure.

Regression analysis and Bland-Altman plots (Figure 1) suggested a systematic underestimation with the wrist monitor across all SBP and DBP measurements. Therefore the readings were adjusted by adding 5 and $10 \mathrm{mmHg}$ to the SBP and DBP records. The adjusted values were plotted and the degree of differences recalculated, resulting in an improvement with $75 \%$ and $77 \%$ for SBP and DBP of the values being within $10 \mathrm{mmHg}$ (grade B). These proportions were $81 \%$ and $91 \%$ for cumulative percentage within $15 \mathrm{mmHg}$ respectively. When the $\mathrm{W}$ measurement was compared to the average mercury readings, 90 and $97 \%$ of the values were within $15 \mathrm{mmHg}$ respectively which were categorized in grade B and A of the BHS criteria. However the device did not pass AAMI protocol despite adjustment because of significant inter-subject diversity in the readings.

\section{PP impact on the validity}

In order to evaluate the possible sources of variations, correlation tests demonstrated that the higher the PP the greater the difference between the wrist-cuff device and the mercury BP measurement. This relationship was statistically significant for the SHOULDER $(\mathrm{r}=0.51, P=0.006)$ and DESK (rho $=0.41, P=0.03$ ) positions for SBP. There was no such relationship between PP and DBP measurement inaccuracies.

\section{Discussion}

This study compared three different limb positions for the wrist-cuff BP measurements and found that the wrist monitors systematically underestimate BP. Generally, using the desk support position with the wrist at the heart level produced closest values to the mercury BP measurement. Although the device failed in direct validation, adjustment by adding 5 and $10 \mathrm{mmHg}$ to the respective SBP and DBP values resulted in acceptable grades according to the BHS protocol. However there was a marked dispersion in our data that was partially related to the effect of PP, and therefore possibly to arterial stiffness in this high risk population.

Our finding regarding the underestimation with wrist monitors compared to a standard mercury monometer is in 
Table 5 Validation assessment according to BHS and AAMI criteria. Values are percentages

\begin{tabular}{|c|c|c|c|c|c|c|c|c|c|c|c|c|}
\hline \multirow[t]{2}{*}{ Arm position } & \multicolumn{3}{|c|}{ SHOULDER } & \multicolumn{3}{|c|}{ HORIZONTAL } & \multicolumn{3}{|c|}{ DESK } & \multicolumn{3}{|c|}{ DESK (adj) } \\
\hline & $\leq 5$ & $\leq 10$ & $\leq 15$ & $\leq 5$ & $\leq 10$ & $\leq 15$ & $\leq 5$ & $\leq 10$ & $\leq 15$ & $\leq 5$ & $\leq 10$ & $\leq 15$ \\
\hline $\begin{array}{l}\text { Average mercury } \\
\text { (SBP) }\end{array}$ & 0 & 23 & 59 & 22.5 & 65 & 70 & 56 & 73.5 & 82 & 65 & 82 & 90 \\
\hline $\begin{array}{l}\text { Average mercury } \\
\text { (DBP) }\end{array}$ & 9 & 24 & 45 & 17.5 & 40 & 65 & 23.5 & 41 & 80 & 31 & 43 & 97 \\
\hline Each mercury (SBP) & 10 & 34 & 56 & 37.5 & 62.5 & 67.5 & 56 & 71 & 76.5 & 60 & 75 & 81 \\
\hline Each mercury (DBP) & 10 & 21 & 44 & 15 & 40 & 62 & 27 & 56 & 74 & 47 & 77 & 91 \\
\hline
\end{tabular}

Abbreviations: AAMI, US Association for the Advancement of Medical Instrumentation; BHS, British Hypertension Society; DPB, diastolic blood pressure; SBP, systolic blood pressure.
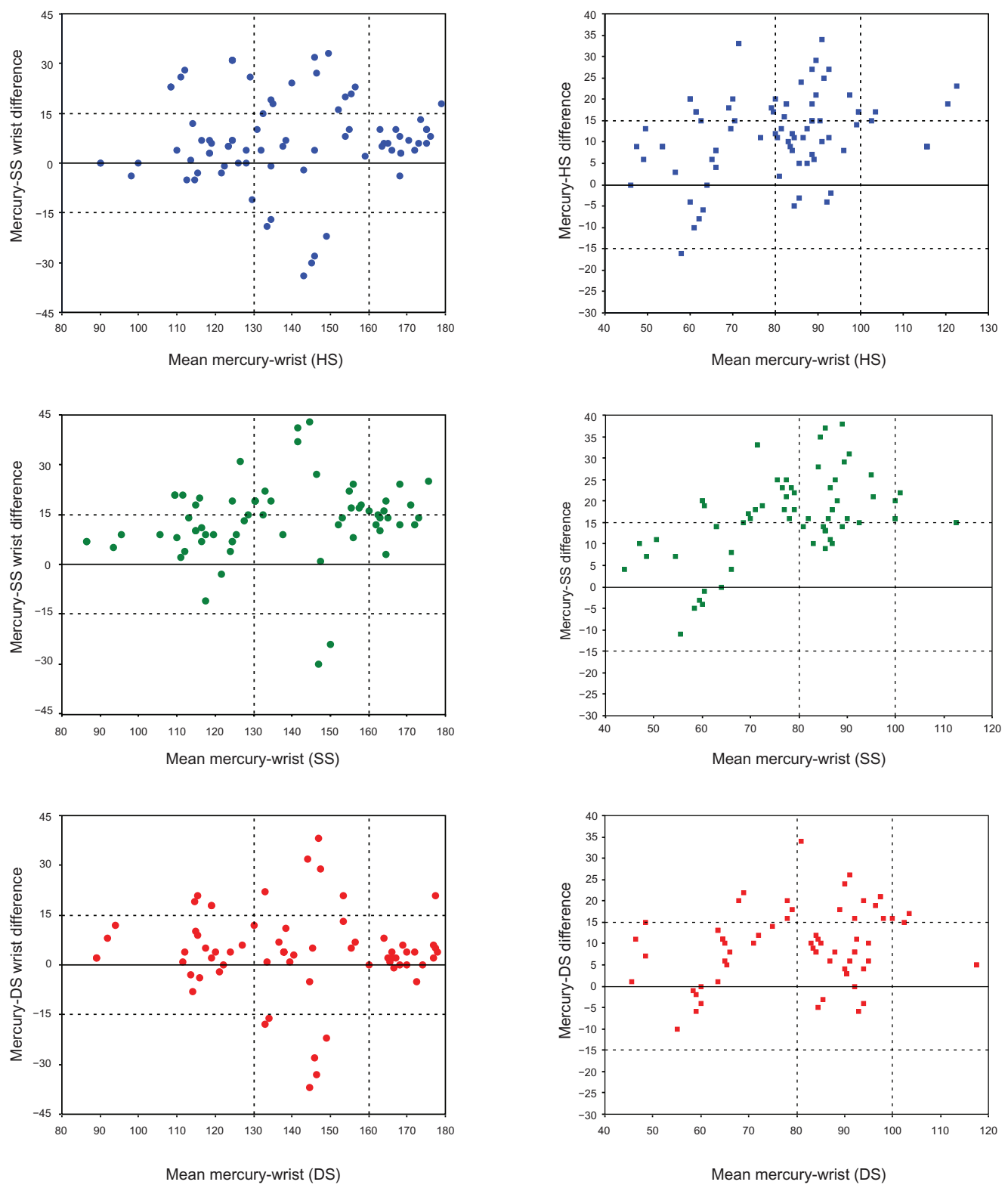

SBP

\section{DBP}

Figure I Bland-Altman plots of blood pressure (BP) measurements with a wrist-cuff monitor with diverse arm positions. Left: SBP; Right:DBP; From top to bottom: horizontallysupported (HS), shoulder-supported (SS), and desk-supported (DS) positions. Abbreviations: DPB, diastolic blood pressure; SBP, systolic blood pressure. 
agreement with the study of Caridi and colleagues ${ }^{12}$ and $\mathrm{El}$ Assaad and colleagues. ${ }^{8}$ However it contradicts others in which an overestimation of BP was observed with a wrist monitor when compared to an upper arm oscillometric device..$^{5,13-15}$ However, this difference may relate to diverse limb positions for wrist-cuff BP measurement, a factor that has not adequately cited in previous reports. Our findings also oppose some previous reports approving the validity of wrist monitor measurements. ${ }^{5,16,17}$ This diversity may be due to different study groups since our group was averagely older than theirs and was from a high risk population. Nevertheless, our study group has more similarity with the average patients in routine clinics and consequently has better clinical applicability than studies on healthy individuals. We observed better validity and less dispersion for DBP measurement compared to SBP with the wrist-cuff device which is supported by previous studies. ${ }^{16,17}$

Although the mercury device is traditionally considered as the gold standard, its accuracy is not clearly identified in high risk population. Watson and colleagues compared both wrist and mercury BP measuring devices with a direct intra-arterial BP measurement in a group of catheterization candidates. They suggested that the wrist monitor was more accurate than the mercury device. ${ }^{18}$ Therefore the systematic underestimation observed with the wrist monitor in our study could have a originated from inaccuracy of the mercury device, rather than the wrist monitor, at least in high risk population. It could be supposed that in patients with significant atherosclerosis or stiff arteries the sound of pulse transmits more rapidly compared to the pulse itself. Consequently one may hear the Korotkoff sound significantly earlier than the travelling pulse. ${ }^{19}$ Also using arm cuffs in patients with obesity, loose fatty arm or conical shape upper arm leads to an overestimation of BP and consequently a difference between mercury and wrist devices.

Many epidemiologic studies used arm-cuff oscillometric $\mathrm{BP}$ values for analyses purposes. Therefore, while wrist-cuff devices are replacing arm-cuff oscillometric monitors, it is more reasonable to compare them with arm-cuff oscillometric monitors rather than a mercury column device. Such a comparison has already been reported by our department indicating a close relationship between the two methods with appropriate arm position. ${ }^{20}$

The results also showed a significant variation in $\mathrm{BP}$ values with the wrist monitor, which was a barrier in achievement of AAMI criteria. This was in parallel with the results of Kikuya and colleagues who found a greater SD for wrist-cuff measurements compared to the upper arm devices. ${ }^{7}$ The possible explanations for this diversity include various degree of arterial stiffness independent of BP. This assumption is supported by the relationship of measurement disagreement with PP, an indirect measure of arterial stiffness in the present study. Furthermore wrist anatomy, incomplete occlusion of radial artery, ${ }^{7,21}$ imprecise limb position due to diversity of intrathoracic heart position and lying, the length and angle of forearm in the case of the shoulder-supported position, wrist anatomy, ${ }^{2,6}$ and finally inappropriate limb angle in particular in obese patients or females with a big upper trunk may also explain such a diversity.

While keeping wrist at heart level is a consensus among all recommendations for BP measurements by wrist monitors, there is little agreements about the limb position in which proper wrist position could be provided. Apart from our previous study, ${ }^{20}$ the only previous report in literature compared sitting with lying position for BP measurement by wrist monitor, reporting remarkably higher values in lying position with wrist at bed level. ${ }^{12}$ In this study we concluded that different limb positions may result in different readings even when the heart level is considered in all positions. Although DESK position was the most accurate one, BP was still underestimated significantly by about $5-10 \mathrm{mmHg}$.

While ambulatory BP monitoring and home selfmeasurement is growing in clinical importance, oscillometric devices are not only devoid of observer-dependency but also allow long-term monitoring, which is not easily achieved by a mercury manometer. Hence, precise readjustment of the oscillometric calculation methods may facilitate their clinical application in selected groups of patients. The cuff oscillometric methods are based on detection of mean arterial pressure and essentially can not directly provide SBP and DBP. They could be calculated by algorithms modified based on a comparison with Korotkoff sounds by manufacturers. ${ }^{7}$ Therefore in any specific group such as elderly or patients with stiff arteries the needed algorithms should be specifically developed..$^{22}$ The proposed device may have a calibration button based on three degrees of arterial stiffness which can be individually set for patients.

Finally, while mercury is eradicated from clinical practice, it is necessary to substitute different, reliable technology as a gold standard for BP measurement. The new technology would not be required to convert the obtained pressure by the oscillometric devices to a mmHg scale and the standard pressure scales could directly be used as a measure of BP. ${ }^{1}$

\section{Conclusion}

Despite the strong correlation between the wrist (Omron HEM 906) and the mercury BP readings, direct values 
obtained by wrist BP monitors are invalid unless they are adjusted. However DESK position yields the closest reading compared to the mercury gold standard and adjusted values with this position attained required criteria for BHS accreditation. From a practical perspective, arterial assessment may help improvement in validity of wrist-cuff monitors in average patients groups.

\section{Disclosures}

The authors report no conflicts of interest in this work. The abstract of this paper has been published in the HBPRCA proceedings in Hypertension. 2007;49:1470.

\section{References}

1. O'Brien E. Will the millimeter of mercury be replaced by the kilopascal? J Hypertens. 1998;16(3):259-261.

2. O'Brien E, Beevers G, Lip GY. ABC of hypertension: Blood pressure measurement. Part IV-automated sphygmomanometry: self blood pressure measurement. BMJ. 2001;322(7316):1167-1170.

3. de Lusignan S, Thiru K, Meredith K, Majeed A, Johnson P. Measuring blood pressure at the wrist: More comfortable for patients and more convenient for doctors. Public Health. 2000;114(3):165-168.

4. O’Brien E, Waeber B, Parati G, Staessen J, Myers MG. Blood pressure measuring devices: recommendations of the European Society of Hypertension. BMJ. 2001;322(7285):531-535.

5. Cuckson AC, Moran P, Seed P, Reinders A, Shennan AH. Clinical evaluation of an automated oscillometric blood pressure wrist device. Blood Press Monit. 2004;9(1):31-37.

6. Parati G, Asmar RG, Stergiou GS. Self blood pressure monitoring at home by wrist devices: a reliable approach? J Hypertens. 2002;20(4):573-578.

7. Kikuya M, Chonan K, Imai Y, Goto E, Ishii M; Research Group to Assess the Validity of Automated Blood Pressure Measurement Devices in Japan. Accuracy and reliability of wrist-cuff devices for selfmeasurement of blood pressure. J Hypertens. 2002;20(4):629-638.

8. El Assaad MA, Topouchian JA, Darné BM, Asmar RG. Validation of Omron HEM-907 device for blood pressure measurement. Blood Press Monit. 2002;7(4):237-241.

9. O’Brien E, Pickering T, Asmar R, et al; Working Group on Blood Pressure Monitoring of the European Society of Hypertension. Working Group on Blood Pressure Monitoring of the European Society of Hypertension International Protocol for validation of blood pressure measuring devices in adults. Blood Press Monit. 2002;7(1):3-17.
10. Imai Y, Otsuka K, Kawano Y, et al; Japanese Society of Hypertension. Japanese Society of Hypertension guidelines for self-monitoring of blood pressure at home. Hypertens Res. 2003;26(10):771-782.

11. British Hypertension Society. Blood Pressure Measurement: Recommendations of the British Hypertension Society. 2004. Available from: http://www.abdn.ac.uk/medical/bhs/. Accessed on December 10, 2009.

12. Caridi G, Zoccali C, Enia G. Oscillometric wrist devices: comparison with the standard mercury sphygmomanometer and estimate of the "supine error" [In Italian]. G Ital Nefrol. 2003;20(6):589-591.

13. Palatini P, Longo D, Toffanin G, Bertolo O, Zaetta V, Pessina AC. Wrist blood pressure overestimates blood pressure measured at the upper arm. Blood Press Monit. 2004;9(2):77-81.

14. Heise T, Magnusson K, Grobel B. A cross-over evaluation of different methods and devices to measure blood pressure in type 1 diabetes patients with nephropathy. Blood Press Monit. 2000;5(3):175-180.

15. Emerick DR. An evaluation of non-invasive blood pressure (NIBP) monitoring on the wrist: comparison with upper arm NIBP measurement. Anaesth Intens Care. 2002;30(1):43-47.

16. Plavnik FL, Zanella MT. Validation study of an automated wrist monitor, Omron model HEM-608, compared with the standard methods for blood pressure measurement. Arq Bras Cardiol. 2001;77(6):532-540.

17. Altunkan S, Yidiz S, Azer S. Wrist blood pressure-measuring devices: a comparative study of accuracy with a standard auscultatory method using a mercury manometer. Blood Press Monit. 2002;7(5):281-284.

18. Watson S, Wenzel RR, di Matteo C, Meier B, Lüscher TF. Accuracy of a new wrist cuff oscillometric blood pressure device: comparisons with intraarterial and mercury manometer measurements. Am J Hypertens. 1998;11:1469-1474.

19. Sebald DJ, Bahr DE, Kahn AR. Narrowband auscultatory blood pressure measurement. IEEE Trans Biomed Eng. 2002;49(9):1038-1044.

20. Mourad A, Gillies A, Carney S. Inaccuracy of wrist-cuff oscillometric blood pressure devices: an arm position artefact? Blood Press Monit. 2005;10(2):67-71.

21. Anderson UO, Henrikson JH, Jensen G. Source of measurement variation in blood pressure in large-scale epidemiological surveys with follow-up. Blood Press. 2002;11(6):357-365.

22. Laurent $\mathrm{S}$, Boutouyrie $\mathrm{P}$, Asmar R, et al. Aortic stiffness is an independent predictor of all-cause and cardiovascular mortality in hypertensive patients. Hypertension. 2001;37(5):1236-1241.
International Journal of General Medicine

\section{Publish your work in this journal}

The International Journal of General Medicine is an international, peer-reviewed open-access journal that focuses on general and internal medicine, pathogenesis, epidemiology, diagnosis, monitoring and treatment protocols. The journal is characterized by the rapid reporting of reviews, original research and clinical studies across all disease areas.

\section{Dovepress}

A key focus is the elucidation of disease processes and management protocols resulting in improved outcomes for the patient. The manuscript management system is completely online and includes a very quick and fair peer-review system. Visit http://www.dovepress.com/ testimonials.php to read real quotes from published authors. 